\title{
Pathological significance and prognostic implications of heme oxygenase 1 expression in non-muscle-invasive bladder cancer: Correlation with cell proliferation, angiogenesis, lymphangiogenesis and expression of VEGFs and COX-2
}

\author{
TOMOHIRO MATSUO, YASUYOSHI MIYATA, KENSUKE MITSUNARI, \\ TAKUJI YASUDA, KOJIRO OHBA and HIDEKI SAKAI \\ Department of Urology, Nagasaki University Graduate School of Biomedical Sciences, Nagasaki 852-8501, Japan
}

Received July 27, 2015; Accepted October 3, 2016

DOI: $10.3892 / 01.2016 .5416$

\begin{abstract}
Heme oxygenase 1 (HO-1) is a stress-response protein and its expression is associated with malignant potential and poor prognosis in several types of cancer. The present study investigated the association between HO-1 expression levels and the pathological features, clinical outcomes and other associated factors in patients with non-muscle-invasive bladder cancer (NMIBC). HO-1 expression was evaluated using immunohistochemistry in 147 formalin-fixed tissue specimens. The proliferation index, microvessel density, lymph vessel density and expression of cyclooxygenase (COX)-2 and vascular endothelial growth factor (VEGF)-A, -C, and -D were also investigated. Correlations among variables were analyzed by multivariate analysis. Survival was assessed using Kaplan-Meier survival curves and multivariate statistics. HO-1 expression levels in high-grade and pT1 tumors were significantly higher compared with low-grade and pTa tumors, and were correlated with the proliferation index $(\mathrm{P}<0.001)$, lymph vessel density $(\mathrm{P}=0.021)$ and $\mathrm{COX}-2$ expression levels $(\mathrm{P}=0.003)$. The proliferation index and $\mathrm{COX}-2$ expression levels were also identified as independent contributing factors in multivariate models. Kaplan-Meier survival curves associated HO-1 expression with a poor prognosis in metastasis-free $(\mathrm{P}=0.047)$ and cause-specific survival $(\mathrm{P}=0.017)$, but not with urinary tract recurrence $(\mathrm{P}=0.231)$. Furthermore, HO-1
\end{abstract}

Correspondence to: Dr Yasuyoshi Miyata, Department of Urology, Nagasaki University Graduate School of Biomedical Sciences, 1-7-1 Sakamoto, Nagasaki 852-8501, Japan

E-mail: int.doc.miya@m3.dion.ne.jp

Abbreviations: HO, heme oxygenase; COX, cyclooxygenase; VEGF, vascular endothelial growth factor; NMIBC, non-muscle-invasive bladder cancer; MVD, microvessel density; LVD, lymph vessel density; PI, proliferation index

Key words: heme oxygenase 1, non-muscle-invasive bladder cancer, cell proliferation, angiogenesis, lymphangiogenesis, vascular endothelial growth factor, cyclooxygenase-2 expression was identified by multivariate analysis to be a significant predictor for cause-specific survival (hazard ratio, 4.08; 95\% confidence interval, 1.06-15.66; $\mathrm{P}=0.004)$. HO-1 has an important role in the malignant aggressiveness of NMIBC and its expression is associated with cause-specific survival. HO-1-associated activities are regulated by cancer cell proliferation, lymphangiogenesis and COX-2. The results suggest that HO-1 may be a potential therapeutic target and a useful predictive prognostic factor in patients with NMIBC.

\section{Introduction}

Bladder cancer is a common malignancy in elderly individuals, particularly in industrialized countries. The standard treatment for early-stage bladder cancer, including non-muscle-invasive bladder cancer (NMIBC), is transurethral resection. However, these tumors have previously been observed to recur and progress to muscle-invasive phenotypes, which then require a radical cystectomy (1). Furthermore, $\sim 50 \%$ of the patients who receive this procedure may subsequently experience local recurrence and metastasis, which are potentially lethal (2). Early detection of malignant potential is essential, as invasive and non-invasive tumors are treated, monitored and managed in different ways, and $\sim 80 \%$ of patients present with non-invasive tumors at diagnosis (3).

At present, histopathological features, including grade and stage, are used as prognostic markers. In addition, various molecular and biological markers, such as survivin, interleukins, microRNAs, have also been reviewed $(4,5)$. However, there are no clinically definitive markers for NMIBC, as tumor growth, invasion, metastasis and recurrence are regulated by complex underlying mechanisms. Tumor cell proliferation and vasculogenesis are associated with cancer progression and prognosis $(6,7)$, as are various cancer-associated molecules, including cyclooxygenase-2 (COX-2) and vascular endothelial growth factor (VEGF) $(8,9)$.

Heme oxygenase 1 (HO-1) is a microsomal enzyme that catalyzes the first, rate-limiting step in the degradation of heme (10). Although HO-1 has numerous functions under physiological conditions, a previous study demonstrated that it is induced in response to cellular stress and hypoxia (11). 
Notably, HO-1 expression levels are elevated in various types of cancer (12-14) and have been demonstrated to regulate certain cancer-associated molecules $(15,16)$. A previous study reported that HO-1 expression levels are significantly associated with malignant aggressiveness and prognosis in patients with bladder cancer (17). However, the prognostic value of HO-1 expression requires further investigation. Processes such as angiogenesis may be differently regulated in non-invasive and invasive bladder tumors (18).

The aim of the present study was to investigate and characterize the HO-1 expression profile in NMIBC cells, and compare it with the clinicopathological features, progression and outcomes of patients with NMIBC. In addition, the potential roles of HO-1 in other cancer processes, including cell proliferation, angiogenesis and lymphangiogenesis, in addition to its association with the expression levels of VEGF and COX-2, were investigated.

\section{Materials and methods}

Patients and tissue samples. Transurethral resection tissue samples from 147 patients with non-invasive bladder cancer, who were treated at Nagasaki University Hospital (Nagasaki, Japan) between 1993 and 2005, were formalin-fixed and paraffin-embedded, placed onto slides and analyzed. The patient population included 116 men $(78.9 \%)$ and 31 women (21.1\%), and their mean age at diagnosis was 68.6 years (standard deviation, 11.6 years). Patients who had previously received neo-adjuvant therapy were excluded, in addition to patients with carcinoma in situ, as these cases are challenging to analyze using immunostaining. Furthermore, cases of squamous cell carcinoma or adenocarcinoma were excluded. Patients were initially examined using chest radiography, ultrasonography, computed tomography of the urinary bladder and abdomen, and cystoscopy. In addition, computed tomography of the lungs or brain, magnetic resonance imaging, drip-infusion pyelography and bone scans were used as required. Stage and grade were assessed in accordance with the 2002 tumor-node-metastasis (TNM) classification (19) and the 2004 World Health Organization grading system (20). The median duration of follow-up was 51 months, with a range of 2-182 months.

The current study was conducted according to the Helsinki II declaration and was approved by the Ethics Review Committee of Nagasaki University Hospital (Nagasaki, Japan). Written informed consent was obtained from all patients prior to enrollment.

Immunohistochemistry. The following primary antibodies were utilized in the current study: Anti-HO-1 (Enzo Life Sciences Inc., Farmingdale, NY, USA; \#ADI-SPA-896-F; dilution, 1:200); anti-Ki-67 (Dako, Glostrup, Denmark; \#M7240; dilution, 1:100); anti-CD34 (Dako; \#M7145); anti D2-40 (Dako; \#M3619; dilution, 1:50); anti-VEGF-A (Santa Cruz Biotechnology Inc., Dallas, TX, USA; \#sc-152; dilution, 1:120); anti-VEGF-C (Zymed; Thermo Fisher Scientific, Inc., Waltham, MA, USA; \#18-2255; dilution, 1:70); anti-VEGF-D (R\&D systems Europe, Ltd., Abingdon, UK; \#MAB286; dilution, 1:100); and anti-COX-2 (Immuno-Biological Laboratories Co., Ltd., Gunma, Japan; \#18515; dilution, 1:40).
Tissue sections (thickness, $5 \mu \mathrm{m}$ ) were deparaffinized in xylene and rehydrated in graded solutions of ethanol. Antigens for the anti-Ki-67 antibody were retrieved at $121^{\circ} \mathrm{C}$

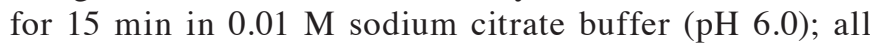
other antigens were retrieved at $95^{\circ} \mathrm{C}$ for $40 \mathrm{~min}$. The tissue sections were immersed in $3 \%$ hydrogen peroxide for $30 \mathrm{~min}$ to block endogenous peroxidase activity. The samples were then probed with the primary antibodies at $4^{\circ} \mathrm{C}$ overnight, then washed with $0.05 \%$ Tween 20 in phosphate-buffered saline. The tissue sections were labeled with peroxidase at room temperature for 60 min using Dako EnVision ${ }^{\mathrm{TM}}$ Peroxidase (Dako; ready-to-use; anti-mouse, \#K4001; anti-rabbit, \#K4003). The peroxidase reaction was visualized with a 3,3'-diaminobenzidine tetrahydrochloride substrate kit (Zymed; Thermo Fisher Scientific, Inc., Waltham, MA, USA; \#00-2014) and the sections were counterstained with hematoxylin. Tumor sections stained with each antibody were examined using a Nikon E-400 bright-field microscope (Nikon, Tokyo, Japan).

A succeeding serial section from each paraffin-embedded tissue sample was processed without primary antibodies as a negative control. Immunohistochemical staining of the positive controls was performed as described previously for all antibodies (6,21-23). Positive control tissues (comprising resected specimens obtained from Nagasaki University Hospital) included samples from colon cancer (VEGF-A, -C and -D), renal cell carcinoma (COX-2), renal vein (CD34) and tonsil (D2-40 and Ki-67). Spleen tissue was used as positive control for HO-1 and the antibodies were used according to the manufacturer's instructions.

Evaluation and interpretation. To evaluate the expression levels of HO-1, immunostained tissue sections were scored semi-quantitatively, as previously described (24). Briefly, the specimens were assigned an immunoreactivity score, which was calculated by multiplying the staining intensity score $(0$, none; 1 , weak; 2 , moderate; 3 , intense) with the score for the density of stained cells $(0,<5.0 \% ; 1,5-25 \% ; 2,26-50 \%$; $3,>51 \%$ ). To determine the microvessel density (MVD) and lymph-vessel density (LVD), the tissue sections were stained with antibodies targeting CD34 and D2-40, respectively. For each tumor section, 3-5 fields with the highest density of stained vessels were evaluated, irrespective of their location in the tumor. MVD and LVD were defined as the number of stained vessels identified in each selected field at x 200 magnification. The proliferation index (PI) was defined as the percentage of cells stained with anti-Ki-67. For these variables, scores greater than the median value were categorized as high, while scores below the median were considered low.

For the expression of VEGFs and COX-2, the staining intensity was graded as none, weak, moderate, or intense; moderate or intense staining was considered a positive reaction. When the percentages of positively stained cancer cells were higher than the median level, the specimen was consider to have high/positive expression for the purpose of survival analyses. Cells were counted in 3-7 randomly selected fields with $\geq 500$ cancer cells.

Slides were examined on a computer-aided image analysis system (Win ROOF version 5.0; Mitani Corporation, Fukui, Japan) and were scored twice at various times by two 
Table I. Association between HO-1 expression levels and clinicopathological features.

\begin{tabular}{|c|c|c|c|c|}
\hline \multirow[b]{2}{*}{ Category } & \multirow[b]{2}{*}{ All patients } & \multicolumn{2}{|c|}{ HO-1 expression } & \multirow[b]{2}{*}{ P-value } \\
\hline & & Negative & Positive & \\
\hline Total number & 147 & 75 & 72 & - \\
\hline Age, years; mean \pm SD & & $66.9 \pm 13.0$ & $70.5 \pm 11.5$ & $0.077^{\mathrm{a}}$ \\
\hline Gender, n (\%) & & & & $0.377^{\mathrm{b}}$ \\
\hline Male & 116 & $57(49.1)$ & $59(50.9)$ & \\
\hline Female & 31 & $18(58.1)$ & $13(41.9)$ & \\
\hline Grade, n (\%) & & & & $0.040^{\mathrm{b}}$ \\
\hline Low & 76 & $45(59.2)$ & $31(40.8)$ & \\
\hline High & 71 & $30(42.3)$ & $41(57.7)$ & \\
\hline pT stage, n (\%) & & & & $0.031^{\mathrm{b}}$ \\
\hline $\mathrm{Ta}$ & 58 & $36(62.1)$ & $22(37.9)$ & \\
\hline $\mathrm{T} 1$ & 89 & $39(43.8)$ & $50(56.2)$ & \\
\hline
\end{tabular}

${ }^{a}$ Analyzed by Student's $t$-test; ${ }^{b}$ analyzed by $\chi^{2}$ test. Samples with percentages of positively stained cancer cells $\leq$ median and $>$ median values were considered negative and positive, respectively. HO-1, heme oxygenase 1; SD, standard deviation; pT stage, pathological tumor stage.

independent pathologists who were blinded to the clinicopathological and survival data.

Statistical analysis. Normally distributed data are presented as the mean \pm standard deviation, whereas the median value and interquartile range are presented for all other data. The Student's $t$-test and the Mann-Whitney U test were used to compare parametric and nonparametric continuous variables, respectively. A $\chi^{2}$-test was used for the categorical comparison of normally distributed data. Survival was assessed using Kaplan-Meier analysis and a log-rank test. Variables that were statistically significant $(\mathrm{P}<0.05)$ in univariate analysis were subsequently subjected to multivariate analysis using Cox proportional hazards, and are reported as odds ratios with $95 \%$ confidence intervals and P-values. All statistical tests were two-sided and were performed using StatView software for Windows (version 5.0; Abacus Concepts, Berkeley, CA, USA). $\mathrm{P}<0.05$ was considered to indicate a statistically significant result.

\section{Results}

HO-1 expression levels and clinicopathological features. In bladder cancer cells, HO-1 was expressed primarily in the cytoplasm (Fig. 1A). HO-1 expression was detected in fibroblast-like, tumor-infiltrating, and endothelial cells; however, there was no specific pattern regarding the distribution of these HO-1-positive cells (Fig. 1B). A total of 72/147 specimens (49.0\%) were determined to have positive expression of HO-1. As indicated in Table I, the number of cells that were positively stained for HO-1 in high-grade tumors $(41 / 71 ; 57.7 \%)$ was significantly higher $(\mathrm{P}=0.040)$ compared with low-grade tumors $(31 / 76 ; 40.8 \%)$. Similarly, a positive association was demonstrated for the pathological tumor $(\mathrm{pT})$ stage $(\mathrm{P}=0.031$; Table I), however, HO-1 expression levels were not associated with patient age at diagnosis $(\mathrm{P}=0.077)$ or gender $(\mathrm{P}=0.377)$.

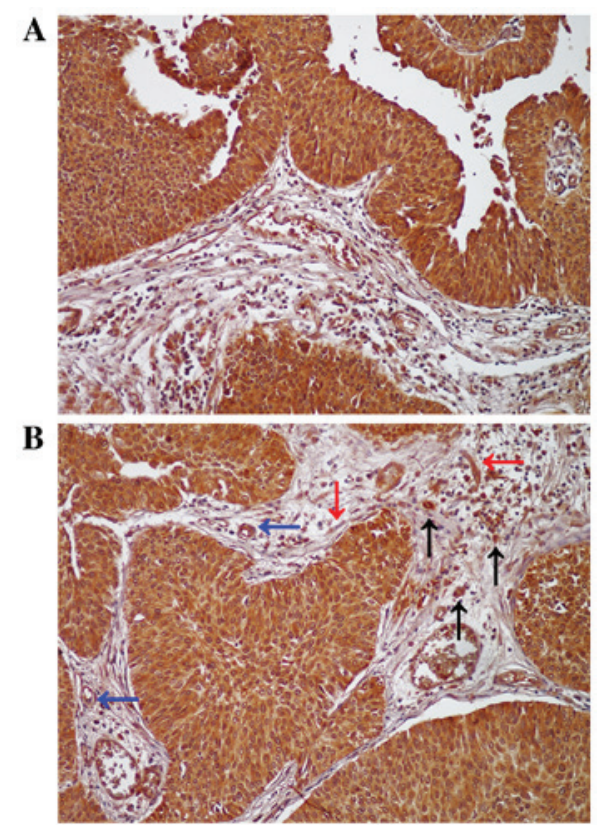

Figure 1. (A) In bladder cancer cells, HO-1 immunostaining was primarily detected in the cytoplasm. (B) In addition to cancer cells, HO-1 immunostaining was present in infiltrating cells (black arrows), fibroblast-like cells (red arrows), and endothelial cells (blue arrows). Magnification, x200. HO-1, heme oxygenase 1 .

HO-1 expression and cancer-associated factors. The association of HO-1 expression levels with MVD, LVD and PI, and the expression profiles of COX-2, VEGF-A, -C, and -D, are depicted in Table II. HO-1 expression levels were associated with COX-2 expression $(\mathrm{P}=0.003)$, LVD $(\mathrm{P}=0.021)$ and PI $(\mathrm{P}<0.001)$, but not with VEGF-A $(\mathrm{P}=0.839)$ or VEGF-C $(\mathrm{P}=0.568)$. HO-1 expression levels were also associated with VEGF-D expression, although this was not determined to be significant $(\mathrm{P}=0.081)$. In a multivariate model that included $\mathrm{pT}$ stage and tumor grade, $\mathrm{HO}-1$ expression was revealed to be 
Table II. Association between HO-1 expression and cancer-associated factors.

\begin{tabular}{|c|c|c|c|c|c|c|}
\hline \multirow[b]{2}{*}{ Category } & \multicolumn{3}{|c|}{ HO-1 expression } & \multicolumn{3}{|c|}{ Multivariate analysis ${ }^{\mathrm{b}}$} \\
\hline & Negative & Positive & P-value ${ }^{a}$ & OR & $95 \% \mathrm{CI}$ & P-value ${ }^{c}$ \\
\hline VEGF-A, \% & $30.8 \pm 13.0$ & $31.2 \pm 12.9$ & 0.839 & - & - & - \\
\hline VEGF-C, \% & $29.4 \pm 14.3$ & $30.7 \pm 13.7$ & 0.568 & - & - & - \\
\hline VEGF-D, \% & $29.6 \pm 12.9$ & $33.3 \pm 13.3$ & 0.081 & - & - & - \\
\hline $\mathrm{COX}-2, \%$ & $18.2 \pm 8.3$ & $22.2 \pm 8.0$ & 0.003 & 2.24 & $1.10-6.13$ & 0.027 \\
\hline MVD, no./HPF & $58.8 \pm 19.9$ & $63.4 \pm 18.0$ & 0.100 & - & - & - \\
\hline LVD, no./HPF & $24.5 \pm 10.3$ & $28.9 \pm 12.4$ & 0.021 & 1.17 & $0.59-2.31$ & 0.659 \\
\hline PI, \% & $19.8 \pm 7.0$ & $24.1 \pm 7.9$ & $<0.001$ & 2.64 & $1.34-5.23$ & 0.005 \\
\hline
\end{tabular}

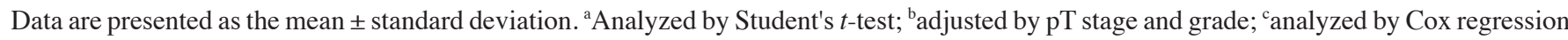
analysis. HO-1, heme oxygenase 1; OR, odds ratio; CI, confidence interval; VEGF, vascular endothelial growth factor; COX-2, cyclooxygenase-2; MVD, microvessel density; LVD, lymph vessel density; PI, proliferation index; pT stage, pathological tumor stage; no./HPF, number per high-power field (x200 magnification).

Table III. Multivariate Cox regression analyses of clinicopathological variables with regard to metastasis and cause-specific survival.

\begin{tabular}{|c|c|c|c|c|c|c|}
\hline \multirow[b]{2}{*}{ Category } & \multicolumn{3}{|c|}{ Metastasis } & \multicolumn{3}{|c|}{ Cause-specific survival } \\
\hline & HR & $95 \% \mathrm{CI}$ & P-value & HR & $95 \% \mathrm{CI}$ & P-value \\
\hline Gender (male vs. female) & 2.88 & $0.37-22.61$ & 0.314 & 1.98 & $0.25-15.77$ & 0.517 \\
\hline pT stage (high vs. low) & 1.32 & $0.40-4.47$ & 0.649 & 2.63 & $0.70-9.93$ & 0.153 \\
\hline Grade (high vs. low) & 4.81 & $1.31-17.69$ & 0.018 & 9.16 & $1.97-42.56$ & 0.005 \\
\hline $\begin{array}{l}\text { Adjuvant treatment (performed } \\
\text { vs. not performed) }\end{array}$ & 2.16 & $0.27-17.07$ & 0.216 & 1.57 & $0.19-12.86$ & 0.673 \\
\hline HO-1 (positive vs. negative) & 2.17 & $0.66-7.11$ & 0.217 & 4.08 & $1.06-15.66$ & 0.040 \\
\hline
\end{tabular}

HR, hazard ratio; CI, confidence interval; pT stage, pathological tumor stage; HO-1, heme oxygenase 1.

independently associated with COX-2 expression $(\mathrm{P}=0.027)$ and $\mathrm{PI}(\mathrm{P}=0.005)$, but not with LVD ( $\mathrm{P}=0.659$; Table II).

HO-1 status and clinical outcomes. The Kaplan-Meier survival curves suggest that HO-1 expression levels were not associated with tumor recurrence in the urinary tract $(\mathrm{P}=0.231$ : Fig. 2A). However, patients with HO-1 positive tumors were identified to have a significantly increased risk of metastasis $(\mathrm{P}=0.047$ : Fig. $2 \mathrm{~B})$ and had poor cause-specific survival ( $\mathrm{P}=0.017$ : Fig. 2C). In addition, $\mathrm{HO}-1$ expression was demonstrated to be a significant and independent predictor of cause-specific survival (hazard ratio, 4.08; 95\% confidence interval, 1.06-15.66; $\mathrm{P}=0.040)$, but not of metastasis, in a multivariate model that included clinicopathological features and adjuvant therapy (Table III).

\section{Discussion}

HO-1 was initially identified as a rate-limiting enzyme in the heme degradation pathway in microsomes (10). In addition to numerous physiological functions, HO-1 has important roles in various pathological conditions, including certain types of cancer. Increased expression levels of HO-1 were previously demonstrated to be associated with malignant potential, cancer cell dissemination and poor prognosis in renal cell, lung and gastric carcinomas $(14,25,26)$. However, other studies have indicated that increased HO-1 expression levels are associated with favorable outcomes in colorectal (27) and tongue cancers (28). Therefore, the pathological significance and prognostic role of $\mathrm{HO}-1$ are considered to be dependent on the type of cancer.

With regard to bladder cancer, a mouse xenograft model of bladder cancer demonstrated that inhibition of HO-1 expression decreased tumor size (29). In human tissues, HO-1 expression was revealed to be a significant marker of tumor recurrence and progression in patients with NMIBC (30). Other studies have also investigated the pathological role of HO-1 expression in patients with NMIBC $(31,32)$; however, there are a number of undetermined features, including the association with vasculogenesis and the regulative mechanisms of HO-1-associated factors. Therefore, the current study focused on the association between HO-1 expression levels and angiogenesis, lymphangiogenesis and vasculogenesis-associated molecules, as these factors are crucial 
A

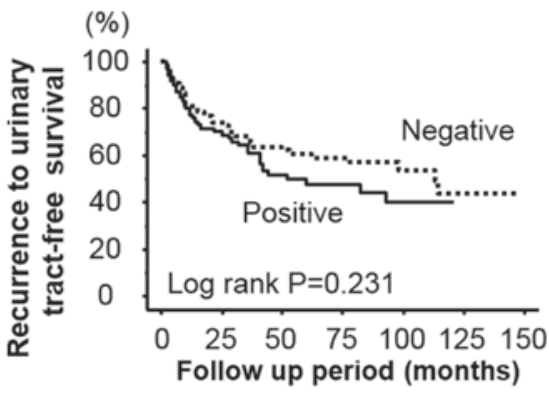

Positive: $\begin{array}{lllllll}72 & 46 & 31 & 16 & 7 & 0\end{array}$

Negative: $\begin{array}{llllll}75 & 51 & 40 & 29 & 15 & 5\end{array}$

Number of patients at risk

B

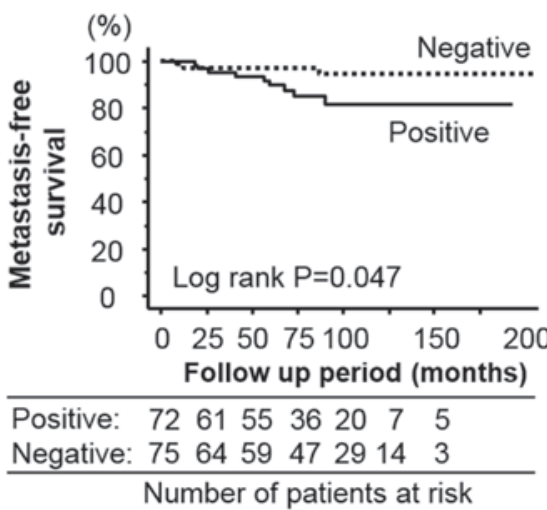

C

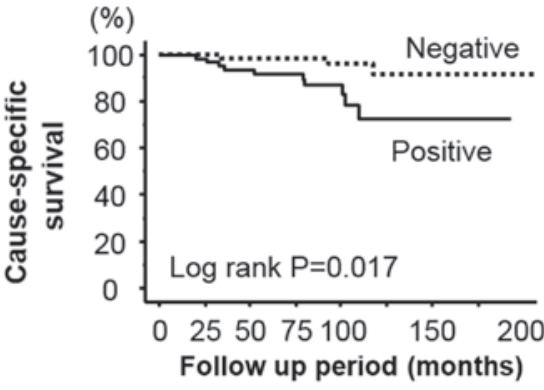

Positive: $7264564021 \quad 75$

Negative: 7566594830143

Number of patients at risk

Figure 2. (A) Kaplan-Meier survival curves indicated that HO-1 expression was not significantly associated with urinary tract tumor recurrence-free survival rates in patients with non-muscle-invasive bladder cancer. However, positive expression of $\mathrm{HO}-1$ was recognized as a predictive factor for poor (B) metastasis-free survival (log rank $\mathrm{P}=0.047)$ and $(\mathrm{C})$ cause-specific survival (log rank $\mathrm{P}=0.017)$. HO-1, heme oxygenase 1 .

for tumor growth, progression and prognosis in bladder cancer (6).

The results of the present study demonstrated that HO-1 expression patterns predict malignant potential and poor prognosis in patients with NMIBC. It was observed that HO-1 expression is associated with tumor grade and $\mathrm{pT}$ stage, concordant with the results of previous studies $(31,32)$. In addition, the data indicate that HO-1 expression is a useful biomarker for progression-free survival, as was previously reported $(31,32)$. To the best of our knowledge, the current study is the first to demonstrate that increased expression levels of HO-1 are significantly associated with poor cause-specific survival in patients with NMIBC.
Cell proliferation, angiogenesis and lymphangiogenesis were assessed in order to investigate the pathological mechanisms underlying the prognostic value of HO-1. The results demonstrated that HO-1 expression levels are significantly associated with cell proliferation, concordant with previous in vivo and in vitro studies $(31,33)$. However, to the best of our knowledge, this study is the first to demonstrate that HO-1 expression levels are correlated with lymphangiogenesis. The association between HO-1 expression and lymphangiogenesis has only previously been investigated in bladder cancer (17), and further studies are required. The current study demonstrated that HO-1 expression levels are not correlated with angiogenesis, contradictory to the results of previous reports (29). This discrepancy may be due to the relatively small sample size $(n=23)$ used in the study, or to the use of various markers to identify the endothelial cells.

Concordant with previous reports (17,34), HO-1 expression is significantly and independently associated with COX-2 expression, which was determined using a multivariate model that included various other pathological features. However, HO-1 expression was not observed to be associated with VEGF expression, as determined by univariate analysis. This result conflicts with the results of previous studies $(17,35)$, which reported that VEGF, particularly VEGF-C and -D, were demonstrated to induce lymphangiogenesis. Therefore, HO-1 may stimulate lymphangiogenesis through mechanisms independent of VEGF signaling.

In conclusion, HO-1 expression is associated with higher grade and pT stage of NMIBC tumors, possibly due to the stimulation of cancer cell proliferation, lymphangiogenesis and COX-2 expression. In multivariate survival analysis, HO-1 expression was identified as a significant predictor for cause-specific survival, but not for metastasis. Therefore, HO-1 has exhibited prognostic value and may be a therapeutic target in patients with early-stage bladder cancer. However, further in vivo and in vitro studies are required to elucidate the underlying mechanisms of HO-1 in NMIBC.

\section{Acknowledgements}

The present study was supported by the Grants-in-Aid for Science Research from the Japanese Society for the Promotion of Science (grant no. 2546487 awarded to Dr Yasuyoshi Miyata; and grant no. 26462415 awarded to Dr Tomohiro Matsuo).

\section{References}

1. Sylvester RJ, van der Meijden AP, Oosterlinck W, Witjes JA, Bouffioux C, Denis L, Newling DW and Kurth K: Predicting recurrence and progression in individual patients with stage Ta T1 bladder cancer using EORTC risk tables: A combined analysis of 2596 patients from seven EORTC trials. Eur Urol 49: 466-477, 2006

2. Cookson MS, Herr HW, Zhang ZF, Soloway S, Sogani PC and Fair WR: The treated natural history of high risk superficial bladder cancer: 15-year outcome. J Urol 158: 62-67, 1997.

3. van Rhijn BW,Burger M,Lotan Y, Solsona E,Stief CG, Sylvester RJ, Witjes JA and Zlotta AR: Recurrence and progression of disease in non-muscle-invasive bladder cancer: From epidemiology to treatment strategy. Eur Urol 56: 430-442, 2009.

4. D'Costa JJ, Goldsmith JC, Wilson JS, Bryan RT and Ward DG: A systematic review of the diagnostic and prognostic value of urinary protein biomarkers in Urothelial bladder cancer. Bladder Cancer 2: 301-317, 2016. 
5. Pospisilova S, Pazourkova E, Horinek A, Brisuda A, Svobodova I, Soukup V, Hrbacek J, Capoun O, Hanus T, Mares J, et al: MicroRNAs in urine supernatant as potential non-invasive markers for bladder cancer detection. Neoplasma 63: 799-808, 2016.

6. Miyata Y, Kanda S, Ohba K, Nomata K, Hayashida Y, Eguchi J, Hayashi $\mathrm{T}$ and Kanetake $\mathrm{H}$ : Lymphangiogenesis and angiogenesis in bladder cancer: Prognostic implications and regulation by vascular endothelial growth factors-A, -C, and -D. Clin Cancer Res 12: 800-806, 2006.

7. Ding W, Gou Y, Sun C, Xia G, Wang H, Chen Z, Tan J, Xu K and Qiang D: Ki-67 is an independent indicator in non-muscle invasive bladder cancer (NMIBC); combination of EORTC risk scores and $\mathrm{Ki}-67$ expression could improve the risk stratification of NMIBC. Urol Oncol 32: 42.e13-e19, 2014.

8. Miyata Y, Watanabe S, Sagara Y, Mitsunari K, Matsuo T, Ohba K and Sakai H: High expression of HuR in cytoplasm, but not nuclei, is associated with malignant aggressiveness and prognosis in bladder cancer. PLoS One 8: e59095, 2013.

9. Wang W, Jiang H, Zhu H, Zhang H, Gong J, Zhang L and Ding Q: Overexpression of high mobility group box 1 and 2 is associated with the progression and angiogenesis of human bladder carcinoma. Oncol Lett 5: 884-888, 2013.

10. Tenhunen R, Marver HS and Schmid R: The enzymatic conversion of heme to bilirubin by microsomal heme oxygenase Proc Natl Acad Sci USA 61: 748-755, 1968

11. Applegate LA, Luscher P and Tyrrell RM: Induction of heme oxygenase: A general response to oxidant stress in cultured mammalian cells. Cancer Res 51: 974-978, 1991.

12. Fang J, Akaike T and Maeda H: Antiapoptotic role of heme oxygenase $(\mathrm{HO})$ and the potential of $\mathrm{HO}$ as a target in anticancer treatment. Apoptosis 9: 27-35, 2004.

13. Lane D, Gray EA, Mathur RS and Mathur SP: Up-regulation of vascular endothelial growth factor- $\mathrm{C}$ by nicotine in cervical cancer cell lines. Am J Reprod Immnol 53: 153-158, 2005.

14. Degese MS, Mendizabal JE, Gandini NA, Gutkind JS Molinolo A, Hewitt SM, Curino AC, Coso OA and Facchinetti MM: Expression of heme oxygenase-1 in non-smal cell lung cancer (NSCLC) and its correlation with clinical data. Lung Cancer 77: 168-175, 2012.

15. Was H,Cichon T,Smolarczyk R,Rudnicka D, Stopa M,ChevalierC Leger JJ, Lackowska B, Grochot A, Bojkowska K, et al: Overexpression of heme oxygenase- 1 in murine melanoma: Increased proliferation and viability of tumor cells, decreased survival of mice. Am J Pathol 169: 2181-2198, 2006.

16. Ferrando M, Gueron G, Elguero B, Giudice J, Salles A, Leskow FC, Jares-Erijman EA, Colombo L, Meiss R, Navone N, et al: Heme oxygenase 1 (HO-1) challenges the angiogenic switch in prostate cancer. Angiogenesis 14: 467-479, 2011.

17. Miyata Y, Kanda S, Mitsunari K, Asai A and Sakai H: Heme oxygenase-1 expression is associated with tumor aggressiveness and outcomes in patients with bladder cancer: A correlation with smoking intensity. Transl Res 164: 468-476, 2014.

18. Kopparapu PK, Boorjian SA, Robinson BD, Downes M Gudas LJ, Mongan NP and Persson JL: Expression of VEGF and its receptors VEGFR1/VEGFR2 is associated with invasiveness of bladder cancer. Anticancer Res 33: 2381-2390, 2013.

19. Sobin DH and Wittekind CH (eds): TNM Classification of Malignant Tumours, 6th edition. Wiley-Liss, New York, pp 199-202, 2002

20. Sauter G, Algaba F, Amin MB, Busch C, Cheville J, Gasser T, Grignon DJ, Hofstädter F, Lopez-Beltran A and Epstein JI; Non-invasive urothelial tumours. In: World Health Organization Classification of Tumours: Pathology and Genetics of Tumours of the Urinary System and Male Genital Organ. Eble JN, Sauter G, Epstein JI, Sesterhenn IA (ed.). IARC Press, Lyon, France, pp 110-123, 2004
21. Miyata Y, Kanda S, Ohba K, Nomata K, Eguchi J, Hayashida Y and Kanetake $\mathrm{H}$ : Tumor lymphangiogenesis in transitional cell carcinoma of the upper urinary tract: Association with clinicopathological features and prognosis. J Urol 176: 348-353, 2006.

22. Miyata Y, Koga S, Kanda S, Nishikido M, Hayashi T and Kanetake H: Expression of cyclooxygenase-2 in renal cell carcinoma: Correlation with tumor cell proliferation, apoptosis, angiogenesis, expression of matrix metalloproteinase-2, and survival. Clin Cancer Res 9: 1741-1749, 2003.

23. Miyata Y, Kanda S, Nomata K, Hayashida Y and Kanetake H: Expression of metalloproteinase-2, metalloproteinase-9, and tissue inhibitor of metalloproteinase-1 in transitional cell carcinoma of upper urinary tract: Correlation with tumor stage and survival. Urology 63: 602-608, 2004.

24. Gandini NA, Fermento ME, Salomón DG, Blasco J, Patel V, Gutkind JS, Molinolo AA, Facchinetti MM and Curino AC: Nuclear localization of heme oxygenase-1 is associated with tumor progression of head and neck squamous cell carcinomas. Exp Mol Pathol 93: 237-245, 2012

25. Banerjee P, Basu A, Wegiel B, Otterbein LE, Mizumura K, Gasser M, Waaga-Gasser AM, Choi AM and Pal S: Heme oxygenase-1 promotes survival of renal cancer cells through modulation of apoptosis- and autophagy-regulating molecules. J Biol Chem 287: 32113-32123, 2012.

26. Yin Y, Liu Q, Wang B, Chen G, Xu L and Zhou H: Expression and function of heme oxygenase-1 in human gastric cancer. Exp Biol Med (Maywood) 237: 362-371, 2012.

27. Andrés NC, Fermento ME, Gandini NA, Romero AL, Ferro A, Donna LG, Curino AC and Facchinetti MM: Heme oxygenase-1 has antitumoral effects in colorectal cancer: Involvement of p53. Exp Mol Pathol 97: 321-331, 2014.

28. Yanagawa T, Omura K, Harada H, Nakaso K, Iwasa S, Koyama Y, Onizawa K, Yusa $\mathrm{H}$ and Yoshida H: Heme oxygenase-1 expression predicts cervical lymph node metastasis of tongue squamous cell carcinomas. Oral Oncol 40: 21-27, 2004.

29. Miyake M, Fujimoto K, Anai S, Ohnishi S, Kuwada M, Nakai Y, Inoue T, Matsumura Y, Tomioka A, Ikeda T, et al: Heme oxygenase-1 promotes angiogenesis in urothelial carcinoma of the urinary bladder. Oncol Rep 25: 653-660, 2011.

30. Yim MS, Ha YS, Kim IY, Yun SJ, Choi YH and Kim WJ: HMOX1 is an important prognostic indicator of nonmuscle invasive bladder cancer recurrence and progression. J Urol 185: 701-705, 2011.

31. Miyake M, Fujimoto K, Anai S, Ohnishi S, Nakai Y, Inoue T, Matsumura Y, Tomioka A, Ikeda T, Tanaka N and Hirao Y: Clinical significance of heme oxygenase-1 expression in non-muscle invasive bladder cancer. Urol Int 85: 355-363, 2010.

32. Kim JH and Park J: Prognostic significance of heme oxygenase-1, S100 calcium-binding protein A4, and syndecan-1 expression in primary non-muscle-invasive bladder cancer. Hum Pathol 45: 1830-1838, 2014.

33. Miyake M, Ishii M, Kawashima K, Kodama T, Sugano K, Fujimoto K and Hirao Y: siRNA-mediated knockdown of the heme synthesis and degradation pathways: Modulation of treatment effect of 5-aminolevulinic acid-based photodynamic therapy in urothelial cancer cell lines. Photochem Photobiol 85: 1020-1027, 2009.

34. Lin $\mathrm{H}$, Huang BR, Yeh WL, Lee $\mathrm{CH}$, Huang SS, Lai $\mathrm{CH}$, Lin H and Lu DY: Antineuroinflammatory effects of lycopene via activation of adenosine monophosphate-activated protein kinase- $\alpha 1 /$ heme oxygenase- 1 pathways. Neurobiol Aging 35: 191-202, 2014

35. Lin HH, Chiang MT, Chang PC and Chau LY: Myeloid heme oxygenase-1 promotes metastatic tumor colonization in mice. Cancer Sci 106: 299-306, 2015. 